pulmonary inflammation and our findings would be consistent with impaired epithelial TGF $\beta$ activation in the lungs of these mice. Further studies are required to determine the origin of the cells activating TGF $\beta$ in these lungs.

\section{S124 MACROPHAGE DELETION OF VHL RESULTS IN ALTERNATIVE ACTIVATION AND ENHANCED LUNG FIBROSIS INDEPENDENT OF HIF-1}

\section{doi:10.1136/thx.2010.150946.25}

${ }^{1} \mathrm{M}$ A Gibbons, ${ }^{1} \mathrm{P} M$ Fitch, ${ }^{1} \mathrm{~A}$ MacDuff, ${ }^{1} \mathrm{~A}$ C Mackinnon, ${ }^{1} \mathrm{~S} J$ Forbes, ${ }^{2} \mathrm{~V}$ Aidinis, ${ }^{3} \mathrm{R}$ S Johnson, ${ }^{1} \mathrm{~T}$ Sethi, ${ }^{1} \mathrm{C}$ Haslett, ${ }^{1} \mathrm{~A}$ G Rossi, ${ }^{1} \mathrm{~A} J$ Simpson, ${ }^{1} \mathrm{~S}$ E M Howie, ${ }^{1} \mathrm{~N}$ Hirani. ${ }^{1}$ University of Edinburgh/MRC Centre for Inflammation Research, Edinburgh, UK; ${ }^{2}$ BSRC Flemming, Athens, Greece; ${ }^{3}$ University of California, San Diego, USA

Background Hypoxia-inducible factor (HIF-1) is a master regulator of the cellular hypoxic response and has been implicated in the pathogenesis of inflammatory and fibrotic disease including IPF.

Aims To study the role of hypoxia and HIF-1 activation in macrophages in the i.t. bleomycin-induced lung fibrosis model.

Methods The i.t. bleomycin model was used to study the effect of HIF-1 manipulation in mice. The primary end-point was lung collagen content at day 24 post i.t. bleomycin instillation. The HIF$1 \alpha$ inducer dimethyloxallyl glycine (DMOG) was administered i.p. on days 14, 17 and 21. The role of myeloid-HIF-1 activity in lung fibrosis was determined using mice in which either HIF- $1 \alpha$ or vHL (the dominant negative-regulator of HIF-1 $\alpha$ ) was selectively knocked out of lysosyme $\mathrm{M}$ expressing cells (LysM-Cre-Hif-1 and Cre-LysMvHL). Lung tissue hypoxia was determined using Hypoxyprobe-1TM administered on day 24. Alternative activation status of HIF-1 null and vHL null macrophages was studied in bone-marrow derived cells from LysM-Cre-Hif-1 and Cre-LysM-vHL mice.

Results Pharmacological induction of HIF-1 in the late period of the bleomycin model with i.p. dimethyloxallyl glycine (DMOG) resulted in significantly enhanced lung collagen (mean \pm s.e.m $\mu \mathrm{g} /$ lung) on day 24 compared to controls ( $193 \pm 15$ vs $152 \pm 8, p<0.05$, $\mathrm{n}>7$ per gp). Hypoxyprobe-1 staining in the bleomycin-injured lung revealed hypoxic alveolar macrophages even in areas of lung distant to patches if severe fibrosis, implying a role for hypoxic/HIF-1 expressing alveolar macrophages in lung fibrosis. However, lung collagen content was identical in myeloid-cell Hif- 1 null mice and wild-type litter-mate controls ( $276 \pm 23$ vs $277 \pm 22, n=8$ per gp). In contrast, myeloid-cell vHL-null mice exhibited significantly enhanced lung collagen deposition versus controls (373 \pm 36 vs $282 \pm 54, \mathrm{p}<0.05, \mathrm{n}>9$ per gp). Isolated $\mathrm{vHL}-\mathrm{null}$ macrophages exhibited enhanced expression of the alternative activation markers YM-1, mannose receptor, arginase-1 and FIZZ-1.

Conclusions vHL deletion in macrophages enhances alternative activation and promotes lung fibrosis independent of HIF-1.

\section{S125 LY6CHI CIRCULATING MONOCYTES DIRECT ALTERNATIVELY ACTIVATED, PRO-FIBROTIC, LUNG MACROPHAGE REGULATION OF PULMONARY FIBROSIS}

doi:10.1136/thx.2010.150946.26

\begin{abstract}
${ }^{1} \mathrm{M}$ A Gibbons, ${ }^{2} \mathrm{~A} \mathrm{C}$ MacKinnon, ${ }^{2} \mathrm{P}$ Ramachandran, ${ }^{2} \mathrm{R}$ Duffin, ${ }^{2} \mathrm{~K}$ Dhaliwal, ${ }^{2} \mathrm{C}$ Haslett, ${ }^{2} \mathrm{~S}$ E M Howie, ${ }^{2} \mathrm{~A} J$ Simpson, ${ }^{2} \mathrm{~N}$ Hirani, ${ }^{3} \mathrm{~J}$ Gauldie, ${ }^{2} \mathrm{~J}$ P Iredale, ${ }^{2} \mathrm{~T}$ Sethi, ${ }^{2} \mathrm{~S} J$ Forbes. ${ }^{1}$ Royal Devon \& Exeter Foundation NHS Trust, Exeter, UK; ${ }^{2} \mathrm{MRC} /$ University of Edinburgh Centre for Inflammation Research, Edinburgh, UK; ${ }^{3}$ Department of Pathology and Molecular Medicine, McMaster University, Hamilton, Canada
\end{abstract}

Introduction and objectives Idiopathic pulmonary fibrosis (IPF) remains one of the few respiratory conditions for which there are no effective therapies. The role of monocytes and macrophages in IPF has been disputed as anti-inflammatory therapies produce questionable benefit. Corticosteroids, however, actually induce an alternatively activated, pro-fibrotic, macrophage phenotype. We sought to determine whether monocytes and macrophages play a role in disease pathogenesis in an attempt to explain why current hypotheses and anti-inflammatory therapies have produced limited clinical benefit despite years of research.

Methods Using multiple in vivo depletional strategies, backed up by an adoptive transfer technique, we extensively investigated the role of monocytes and macrophages during lung fibrogenesis. We performed studies on samples from patients with IPF in an attempt to determine the translational importance of our findings.

Results Depletion of lung macrophages during fibrogenesis reduced pulmonary fibrosis as measured by lung collagen $(p=0.0079)$, fibrosis score $(p=0.0051)$, and qPCR for surrogate markers of fibrosis Coll $(\mathrm{p}=0.0083)$ and a-smooth muscle actin $(\mathrm{p}=0.0349)$. There was an associated reduction in expression of markers of alternative macrophage activation, $Y m 1 \quad(p=0.0179)$, and Arginase1. This reduction was confirmed by immunohistochemistry (IHC) for Ym1 $(p=0.0233)$. IHC on lung macrophages from patients with IPF demonstrated the novel finding of expression of the human alternative macrophage marker CD163. Depletion of Ly6C ${ }^{\text {hi }}$ circulating monocytes reduced pulmonary fibrosis $(p=0.0052)$. Adoptive transfer of Ly6C $\mathrm{C}^{\text {hi }}$ BMDMS during fibrogenesis exacerbated pulmonary fibrosis ( $p=0.0304)$. Furthermore, depletion of circulating Ly $6 C^{\text {h }}$ monocytes lead to a subsequent reduction in the number of Ym1positive alternatively activated lung macrophages $(p=0.0310)$ with a concomitant reduction in the expression of Ym1 and Arginase1.

Conclusions We have demonstrated that monocytes and macrophages do modulate pulmonary fibrosis and suggest that Ly6C $\mathrm{C}^{\text {hi }}$ monocytes (possible fibrocyte precursors) are precursors of alternatively activated, pro-fibrotic, lung macrophages. These findings could link the 'inflammatory' and aberrant wound healing hypotheses and explain the lack of effectiveness of corticosteroids in treating IPF. By enhancing our understanding of the pathogenesis of this dreadful disease, our results may enable new therapeutic targets to be developed, facilitate targeted cell-based therapy, and bring hope to one of the longstanding enigmas of respiratory medicine.

\section{Lung infection: a multi-faceted problem S126 MEASURING QUALITY IN PNEUMONIA CARE. THE NORTH WEST ADVANCING QUALITY PROGRAMME 2008-2009}

doi:10.1136/thx.2010.150946.27

${ }^{1} \mathrm{M}$ Woodhead, ${ }^{2} \mathrm{~J}$ Butterworth, ${ }^{3} \mathrm{~L}-\mathrm{A}$ Myers. ${ }^{1}$ Central Manchester NHS Foundation Trust, Manchester, UK; ${ }^{2}$ North West Strategic Health Authority, Manchester, UK; ${ }^{3}$ Premier Inc, USA

As part of an initiative within the North West Strategic Health Authority to improve the quality of care, 'quality markers' (OMs) were measured in all adult admissions with pneumonia in all 24 Acute Trusts in the North West Region for 1 year (discharges from October 2008 to September 2009). Only adults who fulfilled a prescribed definition of 'pneumonia' were included. OMs were taken from a USA initiative and adapted for UK use. Patient identification was based on clinical coding. Data were recorded in each individual Trust and centrally collated.

Combined data from all trusts OMs were recorded with the following frequencies (no in parentheses is number of patients included): Oxygenation assessment within $24 \mathrm{~h}$ or prior to hospital arrival 96.9\% (11 127), blood culture performed in the A \& E prior to initial antibiotic received in hospital $58.5 \%$ (3323), smoking cessation advice/counselling given in $38.1 \%$ (2788), initial antibiotic consistent with local CAP guidelines $80.8 \%(6337)$ and initial antibiotic received within $6 \mathrm{~h}$ of hospital arrival $64.6 \%$ (7889). Over the four 